\title{
VARIACIONES ESTRUCTURALES Y POSICIONALES DE LOS VOCATIVOS EN EL DISCURSO PARLAMENTARIO SEGÚN EL ROL: PRESIDENCIA FRENTE A OPOSICIÓN
}

\author{
BEATRIZ FIGUEREDO FLORES \\ Grupo Ilse
}

\section{INTRODUCCIÓN}

En Figueredo (2014) nos acercamos al tratamiento de la reformulación por parte de los representantes del Gobierno y de la oposición como forma de expresión cortés. En este caso, nos centraremos en otro elemento de expresión de cortesía, el vocativo, al que someteremos a un doble análisis; el primero de ellos, estructural; el segundo, posicional. En ambos casos podremos observar tendencias variables de un rol a otro.

Nuestro corpus, constituido por los debates en torno al estado de la nación (en adelante, DEN), se halla delimitado en dos aspectos: de un lado, hemos tomado como referencia los discursos iniciales de los presidentes del Gobierno y las correspondientes réplicas emitidas por los líderes de la oposición de los grupos parlamentarios tradicionalmente mayoritarios -Partido Socialista y Partido Popular-. Y por otra parte, la segunda demarcación es de tipo cronológico, pues hemos atendido a los debates celebrados en nuestro siglo, hasta $2011^{1}$. Por lo tanto, abarcamos dieciocho discursos totales: los nueve presidenciales emitidos por José María Aznar y José Luis Rodríguez Zapatero y sus réplicas correspondientes por parte de este último y Mariano Rajoy, periodo que abarca tres legislaturas completas (VII, VIII y IX).

\section{AnÁlisis cuantitativo-EStructural}

El primero de los estudios que proponemos sobre el vocativo, elemento retórico-persuasivo (Bañón, 1993: 53), trata del tipo de estructuras empleadas en su formulación por los portavoces de los principales grupos parlamentarios. Para facilitar la exposición de tal tarea, hemos convenido en agru-

${ }^{1}$ Los años en que se convocan elecciones generales se suspende la celebración de estos debates, lo que explica su ausencia en 2000, 2004 y 2008. 
par las marcas apelativas en dos principales categorías, determinadas por su composición simple -si su mención hace referencia a un cargo- o compleja -cuando va dirigida a más de uno-. A su vez, la primera podrá presentar dos variantes en función del carácter individual o colectivo de su referente. Esto, sin embargo, no ocurre con las formulaciones complejas, de carácter inherentemente colectivo. En otras palabras, y a modo de ejemplo, mientras la mención señor presidente, señoras y señores diputados, por su estructura bimembre es compleja y al mismo tiempo colectiva, señor presidente, por el contrario, es simple e individual, en contraposición a la apelación igualmente constituida por un solo elemento pero con colectivización de los destinatarios, señorías.

TABLA 1. Categorias de las fórmulas de apelación utilizadas en nuestro corpus

\begin{tabular}{|c|c|c|c|}
\hline ESTRUCTURAS & \multicolumn{2}{|c|}{ SIMPLES (UN MIEMBRO) } & COMPLEJAS (BIMEMBRE) \\
\hline CARÁCTER & INDIVIDUAL & Colectivo & Colectivo \\
\hline VOCATIVOS & $\begin{array}{l}\text { Sr. (a) presidente/a } \\
\text { (del Congreso) } \\
\text { Señor presidente } \\
\text { Sr. presidente del Gobierno } \\
\text { Señoría } \\
\text { Señor + apellido(s) } \\
\text { ¡Hombre! }\end{array}$ & $\begin{array}{l}\text { Sras y Sres. diputados } \\
\text { Señorias } \\
\text { Sres. del Gobierno } \\
\text { Señores }\end{array}$ & $\begin{array}{l}\text { Sr (a) presidente/a, señorías } \\
\text { Señorías, sr(a) presidente/a } \\
\text { Sr(a) presidente/a, sras. } \\
\text { y sres. Diputados } \\
\text { Sr. Aznar, señorías } \\
\text { Señorías, señor Aznar }\end{array}$ \\
\hline
\end{tabular}

\subsection{Estructuras simples en los DEN}

En la recurrencia a este tipo de construcciones encontramos una significativa desigualdad cuantitativa entre ambos roles. Sin embargo, esto no obsta para que ambas posiciones muestren una marcada preferencia por el empleo de estructuras simples (cfr. tabla 2).

TABLA 2. Recurrencia media en el empleo de vocativos simples y compuestos por roles

\begin{tabular}{|c|c|c|c|}
\hline \multicolumn{2}{|c|}{ PRESIDENCIA } & \multicolumn{2}{c|}{ OPOSICIÓN } \\
\hline Vocativos simples & Vocativos compuestos & Vocativos simples & Vocativos compuestos \\
\hline 24,4 & 4,7 & 32,5 & 2 \\
\hline
\end{tabular}


Como podemos apreciar, la mayor cantidad de vocativos simples ha sido emitida desde la oposición en un promedio de 32,5 ocurrencias por discurso, en tanto en los discursos presidenciales dicha cantidad desciende a 24,4. Esta diferencia se encuentra justificada por el carácter personalizador de las réplicas de los candidatos en la oposición, enfocado a la respuesta de las cuestiones planteadas por el presidente, y, por tanto, dirigido a él a través de numerosas apelaciones que pueden contener carácter individualizador o generalizador, como veremos más adelante.

A continuación nos detendremos en los tipos de formulaciones simples rastreados en las intervenciones de los presidentes Aznar y Rodríguez Zapatero y, a su vez, en las que aparecen en las réplicas correspondientes de los entonces líderes de la oposición Rodríguez Zapatero y Rajoy. De este modo podremos contrastar las tendencias de empleo en los dos roles.

\subsubsection{Desde el poder}

Según los hallazgos extraídos de nuestro corpus en las emisiones presidenciales de Aznar y Rodríguez Zapatero existen dos tendencias comunes en el empleo de este tipo de elementos.

Por una parte, hallamos una escasa variedad en su formulación, restringida a tres mismas estructuras de tipo salutatorio-honorativo ${ }^{2}$ (Bañón, 1993: 23). La primera fórmula se encuentra dirigida a la persona que en cada momento presidía el Congreso, Señor/a presidente/a, de preferencia menor en Rodríguez Zapatero. De este modo, si Aznar se dirigió a la entonces presidenta del Congreso en algo más de 9 ocasiones por discurso, el gobernante socialista lo hizo en un promedio de 1,5. Otra divergencia entre ambos consiste en la recurrencia a la construcción colectiva señoras y señores diputados, de mayor presencia en las intervenciones de Rodríguez Zapatero (cfr. tabla 4).

Por otro lado, es igualmente significativa la marcada preferencia de los dos presidentes por la estructura colectiva señorías. Esto ocurre de modo especial en el caso del socialista, en una frecuencia media de 23,3 (cfr. tabla 4). Sin embargo, en Aznar el promedio es considerablemente inferior, aunque destaca sobre el resto de apelaciones (cfr. tabla 3).

\footnotetext{
${ }^{2}$ Este término constituye una de las siete categorías funcionales a las que puede pertenecer cualquier vocativo que se incluya en estructuras enunciativas independientes. Sin embargo, la denominación salutatorius aplicada a este elemento apelativo se remonta al mismo Prisciliano, como señala Bañón (1993: 23).
} 
TABLA 3. Estructuras simples en el presidente Aznar

\begin{tabular}{|l|l|c|c|c|c|}
\hline Estructuras & \multicolumn{1}{|c|}{ Vocativos } & 2001 & 2002 & 2003 & PROMEDIO \\
\hline \multirow{3}{*}{ Simples } & Señora presidenta & 6 & 13 & 9 & 9,3 \\
\cline { 2 - 6 } & Señoras y señores diputados & 1 & 1 & 0 & 0,3 \\
\cline { 2 - 6 } & Señorias & 14 & 13 & 9 & 12 \\
\hline \multirow{2}{*}{ TOTAL } & & 21 & 27 & 18 & \multicolumn{2}{|c}{} \\
\hline
\end{tabular}

TABLA 4. Estructuras simples en el presidente Rodríguez Zapatero

\begin{tabular}{|l|l|c|c|c|c|c|c|c|}
\hline Estructuras & \multicolumn{1}{|c|}{ Vocativos } & 2005 & 2006 & 2007 & 2009 & 2010 & 2011 & PROMEDIO \\
\hline \multirow{3}{*}{ Simples } & Señor presidente & 7 & 0 & 0 & 0 & 1 & 1 & 1,5 \\
\cline { 2 - 10 } & $\begin{array}{l}\text { Señoras y señores } \\
\text { diputados }\end{array}$ & 0 & 0 & 0 & 5 & 2 & 0 & 1,16 \\
\cline { 2 - 10 } & Señorías & 24 & 28 & 29 & 21 & 19 & 19 & 23,3 \\
\hline TOTAL & & 31 & 28 & 29 & 26 & 22 & 20 & \multicolumn{2}{|c}{} \\
\cline { 2 - 9 } & & & & & &
\end{tabular}

\subsubsection{Desde la oposición}

Desde la oposición hallamos un considerable aumento en la diversidad de formulaciones apelativas con respecto a las registradas en la presidencia. Esto ocurre por el carácter restringido de las réplicas, cuyos temas se hallan supeditados a la exposición inicial de la presidencia, por lo que la enunciación discursiva se dirige en primera instancia al emisor del discurso precedente, esto es, el presidente del Gobierno, o bien a los diputados presentes en el acto parlamentario. En menor medida, sin embargo, se encuentran las apelaciones a la presidencia del Congreso, mientras que podemos caracterizar como anecdóticas por su aparición las menciones individualizadas a determinados miembros del Parlamento mediante la fórmula señor $/ a+$ apellido.

Planteada esta situación, grosso modo, profundizaremos en las directrices seguidas por los dos líderes de la oposición: Rodríguez Zapatero y Rajoy. De 
la misma manera que vimos cuando comparamos las tendencias generales entre los dos presidentes, en este caso también destacamos un comportamiento diferente para cada uno de los líderes de la oposición, principalmente en el enfoque de sus discursos. Así es como, mientras el líder socialista lo dirige hacia la misma figura del presidente mediante apelaciones constituidas por el tratamiento señor + apellido, Rajoy prefiere destinar sus emisiones al colectivo de miembros del Congreso de diputados a través de su correspondiente colectivo señorías.

\section{Figura 1. Diferencias en el empleo de vocativos en los líderes de la oposición}

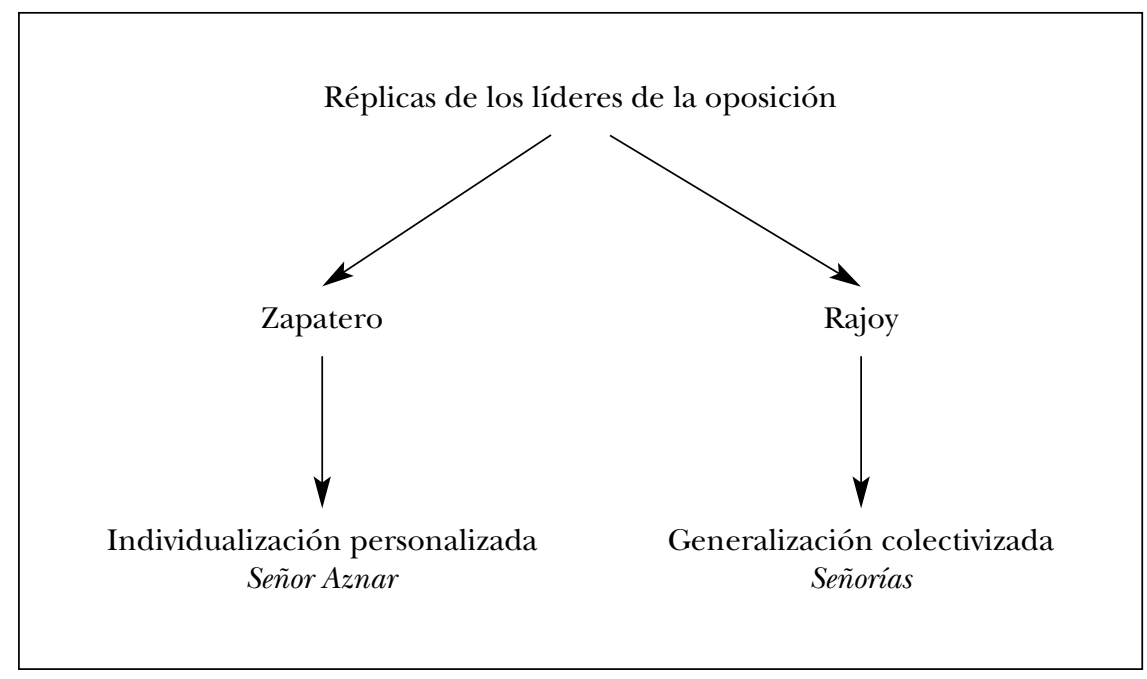

No obstante lo anterior, hemos de destacar una nueva divergencia en la aparición de estas dos formas predominantes, significativamente superior en Rodríguez Zapatero. En efecto, este pronunció la fórmula de tratamiento señor Aznar un total de 93 ocasiones entre 2001 y 2003, o lo que es lo mismo, en un promedio de 31 por intervención. Por su parte, el portavoz del grupo popular utilizó la fórmula colectiva señorías en 108 ocasiones totales, y en un promedio de 18 apariciones por actuación. A pesar de esto, el estudio cuantitativo de formulaciones apelativas simples totales nos ofrece una clara disformidad en la recurrencia de los dos portavoces, pues si el promedio de Rodríguez Zapatero es de 31, el de Rajoy dista 15 unidades de aquel. Estas tablas muestran la variedad de estructuras simples en ambos líderes por años, ordenadas de mayor a menor según la cifra media correspondientes a cada una de ellas (cfr. tablas 5 y 6 ): 
TABLA 5. Estructuras simples en la oposición de Rodríguez Zapatero

\begin{tabular}{|c|c|c|c|c|c|}
\hline Estructuras & Vocativos & 2001 & 2002 & 2003 & PROMEDIO \\
\hline \multirow{10}{*}{ Simples } & Señor Aznar & 17 & 57 & 19 & 31 \\
\hline & Señorias & 9 & 5 & 5 & 6,3 \\
\hline & Señora presidenta & 1 & 1 & 2 & 1,3 \\
\hline & Señor Cascos & 0 & 0 & 3 & 1 \\
\hline & Señor Posada & 1 & 0 & 0 & 0,3 \\
\hline & Señor Rajoy & 1 & 0 & 0 & 0,3 \\
\hline & Señor presidente & 1 & 0 & 0 & 0,3 \\
\hline & Señoría & 0 & 1 & 0 & 0,3 \\
\hline & Señores del Gobierno & 0 & 0 & 1 & 0,3 \\
\hline & ¡Hombre! & 0 & 1 & 0 & 0,3 \\
\hline \multicolumn{2}{|l|}{ TOTAL } & 29 & 65 & 30 & \\
\hline
\end{tabular}

TABLa 6. Estructuras simples en la oposición de Rajoy

\begin{tabular}{|c|c|c|c|c|c|c|c|c|}
\hline Estructuras & Vocativos & 2005 & 2006 & 2007 & 2009 & 2010 & 2011 & MEDIA \\
\hline \multirow{10}{*}{ Simples } & Señorías & 7 & 28 & 10 & 12 & 18 & 33 & 18 \\
\hline & Señoría & 19 & 0 & 6 & 12 & 11 & 1 & 8,16 \\
\hline & Señor Rodríguez Zapatero & 19 & 3 & 7 & 7 & 4 & 2 & 7 \\
\hline & Señor Zapatero ${ }^{3}$ & 1 & 0 & 0 & 0 & 0 & 0 & 0,16 \\
\hline & Señor presidente & 8 & 4 & 20 & 5 & 7 & 2 & 7,66 \\
\hline & $\begin{array}{l}\text { Señor presidente } \\
\text { del Gobierno }\end{array}$ & 0 & 5 & 3 & 0 & 3 & 0 & 1,83 \\
\hline & Señoras y señores diputados & 0 & 2 & 0 & 1 & 0 & 0 & 0,5 \\
\hline & Señora vicepresidenta & 0 & 0 & 1 & 0 & 0 & 0 & 0,16 \\
\hline & Señores & 0 & 0 & 0 & 1 & 0 & 0 & 0,16 \\
\hline & ¡Hombre! & 0 & 0 & 0 & 1 & 0 & 0 & 0,16 \\
\hline \multicolumn{2}{|l|}{ TOTAL } & 54 & 42 & 47 & 38 & 43 & 38 & \\
\hline
\end{tabular}

${ }^{3}$ Nótese la anecdótica forma de referirse al interpelado mediante el tratamiento señor acompañado del segundo apellido, como es habitual referirse a él de forma extraoficial. 
1.1.3. Vocativos simples desde el poder y desde la oposición

De todo lo anteriormente expuesto se deducen las siguientes pautas que podemos establecer en el empleo de este tipo de expresiones apelativas en función del rol desde el que se emitan:

- $\quad$ Mayoritario uso desde la oposición -promedio de 32,5 frente a los 24,4 rastreados en el poder (cfr. tabla 2 )-.

- Generalización de estructuras apelativas de tipo honorativo desde la presidencia, determinadas por el carácter exclusivamente formulista y protocolario de sus emisiones discursivas.

- La propiedad anterior se corresponde con la escasa variedad de fórmulas vocativas, restringidas a las siguientes tres únicas menciones: señor/a presidente/a [del Congreso], señoras y señores diputados y señorias (cfr. tablas 3 y 4 ).

- $\quad$ Por el contrario, por su condición contestataria, las réplicas son más propensas a la personalización de las apelaciones. Esto explica la existencia de una mayor diversidad: desde las formulaciones exclusivas dirigidas al y/o determinado ministro Señor + apellido, Señor presidente, señoría o señor presidente del gobierno hasta las estructuras inclusivas Señores del gobierno, Señoras y señores diputados y señores (cfr. tablas 5 y 6 ).

\subsection{Estructuras compuestas}

Bastante más restringidas en su emisión que las simples, este tipo de construcciones bimembres se limitan casi exclusivamente a las aperturas y cierres de los discursos, lo cual las dotaría de un mayor valor implicativo (Edeso, 2005: 123) de no ser por el carácter estrictamente protocolario del contexto parlamentario, que restringe en gran medida el valor asociado a la cortesía. Como hicimos en el análisis anterior, nos centraremos en las sucesivas exposiciones de las apelaciones registradas en primer lugar en la presidencia, seguidas de sus manifestaciones en los discursos de los líderes de la oposición para acabar con la comparativa entre ambos roles.

\subsubsection{Desde el poder}

Las construcciones dobles se encuentran restringidas a dos tipos en Aznar (cfr. tabla 7) y a tres en Rodríguez Zapatero (cfr. tabla 8). Comunes a ambos son las designaciones a la persona que en cada momento preside 
el Congreso seguidas de la mención al resto de componentes, apelación que puede adoptar dos variantes: el término genérico señorías o bien con mayor especificación de los interlocutores, señoras y señores diputados. La inversión del primer elemento, señorías, señor presidente fue empleado tan solo por el presidente socialista en una única ocasión en el último debate correspondiente al año 2011 (cfr. tabla 8). Con respecto a las otras dos formulaciones, cabe señalar la predilección del presidente conservador por la que implica la generalización del segundo miembro (Señora presidenta, señorias), pronunciada en un total de nueve ocasiones, mientras el gobernante socialista prefiere remitirse al mismo colectivo a través de la estructura señor presidente, señorías en dieciocho ocasiones durante sus seis intervenciones; de otra parte, Aznar recurrió a la fórmula señora presidenta, señoras y señores diputados en un total de seis ocasiones a lo largo de sus tres intervenciones (cfr. tabla 7), mientras en Rodríguez Zapatero tal estructura aparece en catorce ocasiones (cfr. tabla 8).

TABLA 7. Estructuras compuestas en el presidente Aznar

\begin{tabular}{|l|l|c|c|c|c|}
\hline Estructuras & Vocativos & 2001 & 2002 & 2003 & PROMEDIO \\
\hline \multirow{3}{*}{ Compuestas } & $\begin{array}{l}\text { Señora presidenta, señoras } \\
\text { y señores diputados }\end{array}$ & 3 & 1 & 2 & 2 \\
\cline { 2 - 6 } & Señora presidenta, señorias & 1 & 2 & 6 & 3 \\
\hline TOTAL & 4 & 3 & 8 & \multicolumn{2}{|r}{} \\
\cline { 1 - 5 } &
\end{tabular}

TABLA 8. Estructuras compuestas en el presidente Rodríguez Zapatero

\begin{tabular}{|l|l|c|c|c|c|c|c|c|}
\hline Estructuras & \multicolumn{1}{|c|}{ Vocativos } & 2005 & 2006 & 2007 & 2009 & 2010 & 2011 & PROMEDIO \\
\hline \multirow{5}{*}{ Compuestas } & $\begin{array}{l}\text { Señor presidente, señoras } \\
\text { yeñores diputados }\end{array}$ & 6 & 1 & 1 & 1 & 4 & 1 & 2,3 \\
\cline { 2 - 10 } & Señor presidente, señorias & 0 & 0 & 12 & 5 & 0 & 1 & 3 \\
\cline { 2 - 10 } & Señorias, señor presidente & 0 & 0 & 0 & 0 & 0 & 1 & 0,16 \\
\hline \multicolumn{2}{|l|}{ TOTAL } & 6 & 1 & 13 & 6 & 4 & 3 & \multicolumn{2}{|c}{} \\
\hline
\end{tabular}

\subsubsection{Desde la oposición}

Su mínima proporción como fórmula exclusiva de apertura y cierre discursivo -un máximo de dos por discurso- no impide que en ocasiones 
excepcionales nuestros políticos se concedan la licencia de introducir alguno entre las secuencias de desarrollo, excepcional circunstancia común en ambos líderes. En efecto, el portavoz socialista se dirigió al presidente del Gobierno y a los diputados presentes con inversión de sus componentes -señor Aznar, señorías y señorías, señor Aznar- en una ocasión en 2003 durante el progreso argumental de su ponencia (cfr. Tabla 9), en tanto Rajoy hizo lo propio en dos ocasiones en 2005 y una en 2006, 2009 y 2011 con la fórmula de tratamiento Señor presidente, señorías (cfr. tabla 10).

Sin embargo, como ya hemos dicho, lo habitual es la inclusión de tales apelaciones como apertura y cierre discursivo, aunque entre los dos portavoces hallamos diferencias en su empleo. En efecto, esta fórmula es más común en Rajoy que en su predecesor socialista, quien únicamente dedicó en una ocasión la estructura formal Señora presidenta seguida de señorías al inicio de su emisión en 2001 y su inversión Señorías, señora presidenta al final de su discurso el año siguiente. Por el contrario, el líder conservador mantiene tal formulismo en los inicios y cierres de sus discursos casi todos los años exceptuando 2007 y 2010, años en que no se registra ninguna construcción de este tipo (cfr. tablas 9 y 10).

TABLA 9. Estructuras compuestas en la oposición de Rodríguez Zapatero

\begin{tabular}{|l|l|c|c|c|c|}
\hline Estructuras & Vocativos & 2001 & 2002 & 2003 & MEDIAS \\
\hline \multirow{4}{*}{ Compuestas } & Señora presidenta, señorías & 1 & 0 & 0 & 0,3 \\
\cline { 2 - 7 } & Señorías, señora presidenta & 0 & 1 & 0 & 0,3 \\
\cline { 2 - 7 } & Señor Aznar, señorías & 0 & 0 & 1 & 0,3 \\
\cline { 2 - 7 } & Señorías, señor Aznar & 0 & 0 & 1 & 0,3 \\
\hline TOTAL & 1 & 1 & 2 & \multicolumn{2}{|c}{} \\
\hline
\end{tabular}

TABLA 10. Estructuras compuestas en la oposición de Rajoy

\begin{tabular}{|c|c|c|c|c|c|c|c|c|}
\hline Estructuras & Vocativos & 2005 & 2006 & 2007 & 2009 & 2010 & 2011 & MEDIAS \\
\hline \multirow{3}{*}{ Compuestas } & Señor presidente, señorias & 2 & 1 & 0 & 1 & 0 & 1 & 0,83 \\
\hline & Señorías, señor presidente & 0 & 1 & 0 & 0 & 0 & 0 & 0,16 \\
\hline & $\begin{array}{l}\text { Señor presidente, señoras } \\
\text { y señores diputados }\end{array}$ & 0 & 0 & 1 & 0 & 0 & 0 & 0,16 \\
\hline \multicolumn{2}{|l|}{ TOTAL } & 2 & 2 & 1 & 1 & 0 & 1 & \\
\hline
\end{tabular}




\subsubsection{Desde el poder y desde la oposición}

A la luz de los datos expuestos más arriba, podemos destacar en este particular el predominio de apelaciones dobles en las emisiones discursivas pronunciadas desde el poder sobre las empleadas por la oposición. Este hecho, tan lógico como esperable, viene determinado por la opuesta naturaleza de cada uno de estos roles, combativo en el caso de la oposición frente al empeño por la creación de una imagen positiva por parte del Gobierno, para lo cual se recurrirá a una serie de estrategias de cortesía entre las que se encuentran estos elementos. Concretamente, las estructuras compuestas por dos miembros contribuyen de un modo más eficaz a este propósito por implicar un mayor formulismo y por lo tanto expresar una mayor cortesía hacia los interlocutores. En la primera tabla que expusimos al comienzo del presente artículo, dedicada a la comparativa de las cantidades medias de estructuras simples y compuestas por roles, pudimos ver cómo a pesar del predominio del primer tipo en la oposición (32,5 frente a 24,4 desde la presidencia), en el caso de los vocativos compuestos ocurre lo contrario, esto es, la preeminencia de estas fórmulas en las intervenciones de los gobernantes (4,7 frente a 2 desde la oposición) (cfr. tabla 2).

\section{ANÁLISIS ENUNCIATIVO-POSICIONAL}

En este caso, como la denominación del presente epígrafe indica, examinaremos los vocativos hallados en nuestro corpus según el lugar que ocupen en el enunciado, para lo cual aplicaremos la tipología posicional de Bañón (1993: 32-40). Según este autor, estos mecanismos de apelación pueden ocupar dos lugares fundamentales ${ }^{4}$ en la cadena enunciativa: central o marginal, categorías que a su vez contemplan otras subdivisiones secundarias dependiendo de su ubicación con respecto a cada una de ellas (cfr. tabla 11).

Como es fácil de deducir, un vocativo será calificado de central siempre y cuando se sitúe "entre dos partes simétricas de un enunciado" (Bañón, 1993: 33). Entre las escasas muestras registradas en los DEN que estudiamos, se encuentra este ejemplo, donde, como podemos apreciar, el vocativo señor Aznar ocupa este lugar intermedio:

(1) O si no, señor Aznar, cerremos esta Cámara [Zapatero 2001]

\footnotetext{
${ }^{4}$ La acepción del vocablo posición adoptado por Bañón hace referencia a su dimensión sintagmático-lineal (Bañón, 1993: 32). Esta es la que nosotros seguimos en nuestra clasificación.
} 
TABLA 11. Posiciones principales y secundarias de la apelación en el enunciado

\begin{tabular}{|c|c|c|}
\hline POSICIONES PRINCIPALES & Marginal & Central \\
\hline \multirow{4}{*}{ SUBPOSICIONES } & Premarginal & \multirow{2}{*}{ Precentral } \\
\cline { 2 - 2 } & Inicial & \\
\cline { 2 - 2 } & Final & \multirow{2}{*}{ Poscentral } \\
\cline { 2 - 2 } & Posmarginal & \\
\hline
\end{tabular}

Ahora bien, las posiciones pre y poscentrales hacen referencia a lugares inmediatamente precedentes y posteriores al eje enunciativo central, respectivamente. Cabe destacar que mientras la última categoría brilla por su ausencia en nuestro corpus, tan solo hemos hallado un único registro de vocativo precentral ${ }^{5}$ entre los discursos presidenciales y cuatro en las réplicas emitidas desde la oposición:

(2) Está usted reñido, señor presidente, con la claridad, con la transparencia y con el saber a qué atenerse [Rajoy 2010]

Los vocativos marginales, ubicados en los extremos iniciales o finales ${ }^{6}$ del enunciado, pueden presentar dos variantes derivadas cada una

${ }^{5}$ No obstante lo anterior, hemos de aclarar que en muchos casos se han encontrado neutralizaciones entre esta posición y la posmarginal, en cuyos casos, siguiendo el mismo criterio del autor, nos hemos decantado por el término más alejado, y en consecuencia, más distintivo, el posmarginal (Bañón, 1993: 33). Esto explica en parte la abundancia de vocativos pertenecientes a esta última categoría que tendremos ocasión de presentar más adelante.

${ }^{6}$ El caso de los vocativos finales merece igualmente una aclaración previa, puesto que tal posición, de inequívoca y sencilla localización en la cadena enunciativa simple, en estructuras complejas el criterio clasificatorio-posicional puede hacerse en términos absolutos o en términos relativos (Bañón, 1993: 40). La categoría absoluta es equivalente a la posición ocupada en el enunciado simple, esto es, constituyendo el término del enunciado:

(i) Es imposible que complete las reformas quien ni siquiera tiene conciencia de que están incompletas, ni con elecciones ni sin elecciones, señorías [Rajoy 2011]

Sin embargo, cuando el vocativo se sitúa tras una enunciación completa desde el punto de vista sintáctico -esto es, formada por al menos un sintagma verbal- aparece seguido de una construcción compleja -bien sea en relación de yuxtaposición, coordinación o subordinación-, hablamos de vocativo en posición final relativa:

(ii) Esto es como la guitarra, señorías: o se afinan las seis cuerdas de la guitarra o la guitarra sigue desafinada [Rajoy 2011]

(iii) Nada de eso crea empleo, señorías, y lo que no crea empleo ya cansa [Rajoy 2009]

(iv) Le quiero decir, señor Aznar, que hoy España está más aislada que nunca en Europa desde la democracia [Zapatero 2001] 
de las dos anteriores: posmarginales si se halla justo tras el margen inicial ${ }^{7}$ y premarginales, en este caso inmediatamente anterior al último elemento de la secuencia enunciativa. Los siguientes ejemplos corresponden a cada una de estas tipologías posicionales, expuestas en estricto orden expositivo -marginal (3) inicial, marginal final (4), posmarginal (5) y premarginal (6)-:

(3) Señorías, nuestra economía sigue en una senda de crecimiento [Aznar 2001]

(4) Voy concluyendo, señorías [Zapatero 2011]

(5) En España, señor Aznar, no hay políticas de atención a los niños de cero a tres años [Zapatero 2003]

(6) Esta vez era un nuevo milagro de usted, señor Aznar, un milagro estadístico [Zapatero 2002]

\subsection{Posición de los vocativos en la presidencia}

El lugar predominante de las fórmulas de apelación en los discursos presidenciales de apertura se encuentra al inicio de la cadena enunciativa. Tanto es así que las medias totales de ambos líderes superan la veintena tanto en Aznar como en Rodríguez Zapatero -exceptuando los 15 de 2011-; son en este caso imperceptiblemente más frecuentes en las intervenciones del presidente conservador $(24,6)$ que en el gobernante socialista, cuya media fue de 23,6 (cfr. tabla 12). Si desglosamos por intervención el número de veces que fue empleado tal elemento, veremos cómo las cantidades varían en Aznar entre un máximo de 26 en 2002 y un míni-

${ }^{7}$ Creemos oportuno matizar también que, dada la conocida complejidad sintáctica de las construcciones discursivas de nuestros políticos en sus emisiones parlamentarias, en muchos casos nos hemos encontrado con vocativos de difícil clasificación posicional, especialmente en el caso de los posmarginales. En estos casos hemos tomado como criterio selectivo la posición precedente del mismo con respecto al verbo:

(i) Sin duda, señorías, es mejor que las cosas se hagan bien, aunque a la fuerza que mal por inclinación [Rajoy 2010]

Sin embargo, en casos muy aislados en los que el inicio del enunciado coincide con una construcción verbal seguida por un vocativo, hemos considerado a este último igualmente como posmarginal, por hallarse ubicado tras el margen inicial:

(ii) Hablo, señorías, de un recelo mesurable que ha crecido al mismo ritmo mesurado desaforado de nuestra deuda [...] [Rajoy 2011] 
mo de 24 en 2001 y $2003^{8}$, mientras que en el líder socialista oscilan entre las 30 en 2007 y las 15 de 2011 (cfr. tabla 13).

Los vocativos finales, de otro lado, aparecen en un promedio de 1 en el gobernante popular -empleado una vez en cada año-, igual que en el socialista, pues las tres ocasiones en las que fue empleado en 2005, las dos en 2007 y la única de 2011 ofrecen el mismo promedio de uno.

El resto de posiciones aparecen en cantidades mínimas en ambos, especialmente la premarginal y precentral -limitadas a una única aparición en Aznar en 2003 en el primer caso y en el presidente socialista en 2010-. Los vocativos centrales, por su parte, fueron únicamente empleados por Zapatero en dos ocasiones en 2005 y una en $2010^{9}$. Como también podemos apreciar en las tablas 12 y 13, la ubicación que brilla por su ausencia en ambos casos es la poscentral.

Con respecto a la posmarginalidad enunciativa cabe indicar que aparece en cuatro ocasiones en el líder conservador, en contraposición a la significativa frecuencia registrada en Rodríguez Zapatero, con un total de 36 repartidas entre 2005 y 2011, cuyo promedio de 6 contrasta notablemente con el 1,3 de Aznar.

(7) Pensamos, señorias, que a los mayores de 52 años hay que darles algún horizonte adicional a la jubilación [Aznar 2002]

(8) Por ello, señorías, hemos recordado a los más olvidados, [...] [Zapatero 2005]

8 A pesar de ser la ocasión en la que el presidente conservador recurrió en menor medida a este recurso, se trata, por otra parte, de casi la única posición registrada en este año. Por lo tanto, en 2001 todas -menos el vocativo en posición final [Muchas gracias] señora presidenta, coincidiendo además con el término de su intervención para agradecer el turno de palabra concedido- las ubicaciones enunciativas en las que Aznar pronunció las apelaciones fueron de tipo marginal inicial.

${ }^{9}$ Las siguientes secuencias extraídas de nuestro corpus son algunos ejemplos de las ocasiones en las que nuestros gobernantes situaron un vocativo en las ubicaciones mencionadas:

(i) El programa que impulso como presidente del Gobierno nunca ha sido, señorías, un proyecto personal [Aznar 2003] (Premarginal)

(ii) En lo tocante al compromiso de Kioto para la reducción de gases de efecto invernadero, señorías, el anterior Gobierno siguió una política a medio camino entre Europa y Estados Unidos [Zapatero 2005] (Central)

(iii) Varios han sido, señorías, los sectores sociales que han recibido una atención singular del Gobierno en este primer año de trabajo [...] [Zapatero 2005] (Posición central relativa)

(iv) Muchas gracias, señora presidenta [Aznar 2002] (Final)

(v) Voy concluyendo, señorías [Zapatero 2010] (Final) 
TABLA 12. Cantidades medias en la aparición de vocativos según su posición en la presidencia: Aznar y Zapatero

\begin{tabular}{|l|l|c|c|}
\hline \multicolumn{2}{|c|}{ POSICIÓN } & AZNAR & ZAPATERO \\
\hline \multicolumn{2}{|c|}{ Premarginal } & 0,33 & 0 \\
\hline \multirow{2}{*}{ Marginal } & Inicial & 24,6 & 23,6 \\
\cline { 2 - 4 } & Final & 1 & 1 \\
\hline Posmarginal & 1,3 & 6 \\
\hline Precentral & 0 & 0,16 \\
\hline Central & 0 & 0,5 \\
\hline Poscentral & 0 & 0 \\
\hline
\end{tabular}

TABLA 13. Cantidades totales de apelaciones según su posición en la presidencia por años

\begin{tabular}{|l|c|c|c|c|c|c|c|c|c|c|}
\cline { 2 - 13 } \multicolumn{2}{c|}{} & \multicolumn{10}{c|}{ PRESIDENCIA } \\
\hline \multicolumn{2}{|c|}{ PosıcIón } & 2001 & 2002 & 2003 & 2005 & 2006 & 2007 & 2009 & 2010 & 2011 \\
\hline \multicolumn{2}{|c|}{ Premarginal } & $\times$ & $\times$ & 1 & $\times$ & $\times$ & $\times$ & $\times$ & $\times$ & $\times$ \\
\hline \multirow{2}{*}{ Marginal } & Inicial & 24 & 26 & 24 & 28 & 28 & 30 & 21 & 20 & 15 \\
\cline { 2 - 13 } & Final & 1 & 1 & 1 & 3 & $\times$ & 2 & $\times$ & $\times$ & 1 \\
\hline Posmarginal & $\times$ & 4 & $\times$ & 5 & 1 & 10 & 7 & 5 & 8 \\
\hline Precentral & $\times$ & $\times$ & $\times$ & $\times$ & $\times$ & $\times$ & $\times$ & 1 & $\times$ \\
\hline Central & $\times$ & $\times$ & $\times$ & 2 & $\times$ & $\times$ & $\times$ & 1 & $\times$ \\
\hline Poscentral & $\times$ & $\times$ & $\times$ & $\times$ & $\times$ & $\times$ & $\times$ & $\times$ & $\times$ \\
\hline
\end{tabular}

(9) España, señorías, acumula superávits consecutivos en sus cuentas públicas desde 2004 [...] [Zapatero 2007]

(10) En definitiva, señoras y señores diputados ${ }^{10}$, me comprometo antes ustedes a impulsar este acuerdo para la recuperación económica y la protección social [...] [Zapatero 2009]

${ }^{10}$ Resulta llamativa que la fórmula empleada para este lugar sea la estructura simple colectiva señorías en casi todas las ocasiones, a excepción de la bimembre señoras y señores diputados que vemos en este ejemplo. 
TABLA 14. Cantidades medias en la aparición de vocativos según su lugar en la oposición: Zapatero y Rajoy

\begin{tabular}{|l|l|c|c|}
\hline \multicolumn{2}{|c|}{ POSICIÓN } & ZAPATERO & RAJOY \\
\hline \multicolumn{2}{|c|}{ Premarginal } & 2 & 0,16 \\
\hline \multirow{2}{*}{ Marginal } & Inicial & 11,6 & 15,6 \\
\cline { 2 - 4 } & Final & 19,6 & 17,8 \\
\hline Posmarginal & 8 & 10,6 \\
\hline Precentral & 1 & 0,16 \\
\hline Central & 0,3 & 0,3 \\
\hline Poscentral & 0 & 0 \\
\hline
\end{tabular}

\subsection{Posición de los vocativos en la oposición}

Si los discursos presidenciales se caracterizan por una dominante presencia de vocativos situados al comienzo de la cadena enunciativa, en las réplicas de los líderes de la oposición la localización dominante se encuentra en su lugar linealmente opuesto dentro de la secuencia hablada, es decir, en el cierre del discurso. No obstante lo anterior, aunque los promedios correspondientes a esta modalidad rebasan significativamente el resto de categorías, no llegan a alcanzar la veintena en ninguno de los portavoces (cfr. tabla 14). Con respecto a las apelaciones situadas al final de la cadena enunciativa hemos de hacer notar la similitud en su empleo medio en los dos líderes, escasamente inferior en Rajoy (17,8 frente a los 19,6 del socialista). Si nos remitimos a los registros hallados por año en cada uno, en este último presentan un valor máximo de 37 en 2002 y un mínimo de 10 en 2001, mientras el rango de frecuencias hallado en Rajoy fluctúa entre 28 en 2005 y 7 en $2009^{11}$ (cfr. tabla 15 ):

(11) Le diré que la situación es insostenible, señor Aznar [Zapatero 2001]

(12) Los precios suben en España, señoría, y la mayoría de las familias españolas pierde poder adquisitivo [Zapatero 2002]

${ }^{11}$ Creemos que no está de más destacar que, aunque en los ejemplos puede verse claramente, bajo la mención a la posición final del vocativo nos referimos tanto a los que se insertan en estructuras oracionales simples y complejas, de un lado, como a los evaluados en términos relativos teniendo en cuenta los criterios expuestos en la introducción de este estudio enunciativo-posicional. 
(13) Esta es la España que está usted construyendo, señoría: así es el progreso que nos ha traído [Rajoy 2005]

(14) Es llamativo, señor presidente [Rajoy 2006]

(15) Esta es la realidad, señorías [Rajoy 2009]

(16) Es evidente, señorías, que la cuestión que debatimos aquí no es si España está bien o está mal [Rajoy 2010]

Como ya hemos indicado, cabe destacar la mayor recurrencia por parte del portavoz conservador en el empleo de un vocativo en situación inicial del discurso, cuyo rango oscila entre las 22 apariciones de 2006 y las 12 registradas en 2005 y 2011. Por su parte, Rodríguez Zapatero limita tal posición a una máxima de 16 en 2002 y 9 en su primera réplica (cfr. tabla 15).

(17) Señora presidenta, señorías, la primera vez que voté en mi vida voté la Constitución española de 1978 [Zapatero 2001]

(18) Señorías, los acontecimientos de la última semana me exigen hacer dos pronunciamientos previos [...] [Zapatero 2002]

(19) Señor presidente, señorías, tras el idílico panorama que nos ha pintado el presidente del Gobierno esta mañana, la realidad es que si un viajero hubiera estado fuera de España durante un año y regresara hoy no daría crédito a lo que ve [Rajoy 2005]

(20) Señorias, he escuchado con mucha atención el discurso del señor Rodríguez Zapatero de esta semana [Rajoy 2006]

(21) Señor presidente, señorías, nunca han hecho falta menos palabras para exponer el estado de la Nación [Rajoy 2009]

(22) Señorías, tras escuchar esta mañana al señor Rodríguez Zapatero, la pregunta que cabe hacerse es: ¿en qué se diferencia este debate del que celebramos hace un año? [Rajoy 2010]

Sin embargo, esto no implica la ausencia de los mismos en las otras secuencias. Los tres últimos ejemplos constituyen los cierres del discurso pronunciado el año que se indica:

(23) Señor Aznar, acabo de recibir una carta de becarios de países sobre todo iberoamericanos, denunciando que la Agencia de cooperación había suprimido el programa de beca para la gente de otros países [Zapatero 2001]

(24) Señor Aznar, estamos a la cola en investigación y desarrollo, estamos a la cola de Europa [...] [Zapatero 2002]

(25) Señor Aznar, se va usted como llegó [Zapatero 2003] 
(26) Señor presidente ¿y qué mas ha hecho usted? [Rajoy 2005]

(27) Señor presidente del Gobierno, ha aumentado el número de delitos [Rajoy 2006]

(28) Señorías, señor presidente -termino definitivamente- [...] [Zapatero 2001]

(29) Señor presidente, señorías, muchas gracias [Rajoy 2005]

(30) Señor presidente [de la Cámara], le agradezco su condescendencia [Rajoy 2007]

Por otra parte, los vocativos posmarginales no destacan por su abundancia en ninguno de los dos líderes, siendo Rodríguez Zapatero quien menor atención les concedió a lo largo de sus emisiones discursivas. Los valores correspondientes a este último se sitúan entre los 10 de 2002 y 5 en 2001, mientras que los propios del conservador se mantuvieron entre 7 en 2010 y 12 en 2007, 2009 y 2011 (cfr. tabla 15).

(31) La educación, señor Aznar, es el instrumento más poderoso de las sociedades modernas para una igualdad real de oportunidades [Zapatero 2002]

(32) Telefónica, señor Aznar, es el mejor ejemplo de cómo su política de privatización [...] es una gran mentira [...] [Zapatero 2003]

(33) Ahora, señorías, dejando ya a un lado todos estos aspectos parciales de la actividad del Gobierno [...] [Rajoy 2006]

(34) En suma, señorías, esto es lo que se debe hacer, amén de otras cosas en las que por razones de tiempo no voy a extenderme [Rajoy 2009]

En otra instancia, las posiciones menos tenidas en cuenta por la oposición son la central, la precentral y la premarginal. La primera hace su aparición en tres ocasiones totales en el caso de los dos portavoces de la oposición (cfr. tabla 15).

(35) O si no, señor Aznar, cerremos esa cámara [Zapatero 2001]

(36) El fin de la cuestión, Señor Rodríguez Zapatero, al servicio de otros intereses [Rajoy 2005]

El vocativo precentral, por su parte, se reduce a tres apariciones en Zapatero y a una en Rajoy (cfr. tabla 15):

(37) ¿Se acuerda, señor Aznar, del plan policía 2000 ? $^{12}$ [Zapatero 2002]

${ }^{12}$ A pesar de lo dudoso de esta ubicación entre las categorías central y precentral, la mayor extensión del complemento verbal que sigue al vocativo con respecto al núcleo verbal precedente, ha hecho que nos decantemos por esta última. 
(38) Está usted reñido, señor presidente, con la claridad, con la transparencia y con el saber a qué atenerse [Rajoy 2010]

Con respecto a la ubicación premarginal cabe indicar que fue empleada por el portavoz liberal en mayor frecuencia que en el portavoz conservador; seis ocasiones totales: 5 en 2001 y 1 en 2003 (cfr. tabla 15):

(39) [...] hemos observado un cambio de talante y de actitud con el nuevo ministro del Interior, señor Rajoy, en esta materia [Zapatero 2001]

(40) Por tanto, en cuestión social y en bienestar del país, con ambición de modernización e innovación estamos, señor Aznar, claramente suspendiendo [Zapatero 2001]

(41) [...] y supongo que aún recuerda a aquellos marineros en las Rías Bajas, luchando con sus manos contra el chapapote y preguntándose dónde estaba su Gobierno, señor Aznar, en aquellos momentos [Zapatero 2003]

TABLA 15. Cantidades totales de apelaciones según su posición en la oposición por años

\begin{tabular}{|l|c|c|c|c|c|c|c|c|c|c|}
\cline { 2 - 12 } \multicolumn{1}{c|}{} & \multicolumn{8}{c|}{ OPOSICIÓN } \\
\hline \multicolumn{2}{|c|}{ POSICIÓN } & 2001 & 2002 & 2003 & 2005 & 2006 & 2007 & 2009 & 2010 & 2011 \\
\hline \multicolumn{2}{|c|}{ Premarginal } & 5 & $\times$ & 1 & $\times$ & $\times$ & 1 & $\times$ & $\times$ & $\times$ \\
\hline \multirow{2}{*}{ Marginal } & Inicial & 9 & 16 & 10 & 12 & 22 & 14 & 18 & 16 & 12 \\
\cline { 2 - 13 } & Final & 10 & 37 & 12 & 28 & 13 & 22 & 7 & 21 & 16 \\
\hline Posmarginal & 5 & 10 & 9 & 11 & 10 & 12 & 12 & 7 & 12 \\
\hline Precentral & $\times$ & 3 & $\times$ & $\times$ & $\times$ & $\times$ & $\times$ & 1 & $\times$ \\
\hline Central & 1 & $\times$ & $\times$ & 1 & $\times$ & $\times$ & 1 & $\times$ & $\times$ \\
\hline Poscentral & $\times$ & $\times$ & $\times$ & $\times$ & $\times$ & $\times$ & $\times$ & $\times$ & $\times$ \\
\hline
\end{tabular}

\subsection{Posición de los vocativos en la presidencia y en la oposición}

Según los resultados mostrados en los epígrafes precedentes, podemos proceder a delimitar unas marcadas pautas a la hora de situar el vocativo en la cadena enunciativa; tales resultados, como hemos comprobado y veremos, variarán en función del rol desde el que sean emitidos. 
En primer lugar, como puede comprobarse en la tabla comparativa que presentamos, el cotejo de la frecuencia de uso por roles determina una significativa propensión hacia la ubicación de este tipo de recursos al comienzo del enunciado por parte de la presidencia. De hecho, la cantidad media es cuantitativamente superior al resto, pues rebasa casi en veinte unidades a la segunda ubicación en orden de frecuencia, correspondiente en este caso a la posmarginal (cfr. tabla 16).

Sin embargo, desde la oposición encontramos dos ubicaciones que destacan sobre las demás: la marginal final $(18,4)$ y la inicial $(14,3)$. Como vemos, a pesar de la coincidente predilección por la posición inicial en ambos roles, el promedio de 14,3 de la oposición está suficientemente superado por los 24 que vimos desde el poder. Ahora bien, esto no se corresponde con lo que ocurre con la posición final, cuyo inapreciable promedio de 1 contrasta significativamente con los 18,4 en el caso de la oposición (cfr. tabla 16).

En referencia a la apelación posmarginal cabe hacer notar su baja reincidencia en ambos roles, aunque el promedio de 9,7 correspondiente a la oposición supone una ventaja de 5,3 sobre el correspondiente al poder. En el resto de lugares no hemos encontrado diferencias significativas, esto es, con respecto a los vocativos centrales, precentrales y poscentrales ( $\mathrm{cfr}$. tabla 16).

TABLA 16. Comparativa de cantidades medias de tipos de apelaciones por rol: presidencia y oposición

\begin{tabular}{|l|c|c|c|}
\hline \multicolumn{2}{|c|}{ POSICIÓN } & PRESIDENCIA & OPOSICIÓN \\
\hline \multicolumn{2}{|c|}{ Premarginal } & 0,1 & 0,7 \\
\hline \multirow{2}{*}{ Marginal } & Inicial & 24 & 14,3 \\
\cline { 2 - 4 } & Final & 1 & 18,4 \\
\hline Posmarginal & 4,4 & 9,7 \\
\hline Precentral & 0 & 0,4 \\
\hline Central & 0,3 & 0,3 \\
\hline Poscentral & 0,1 & 0 \\
\hline
\end{tabular}




\section{BIBLIOGRAFÍA}

Bañón Hernández, A. M. (1993): El vocativo en español. Propuestas para su análisis lingüistico, Barcelona, Octaedro.

Edeso, V. (2005): "Usos discursivos del vocativo en español”, Español Actual, 84, págs. 124-142.

Figueredo Flores, B. (2014): "Otros valores de la aclaración como estrategia en el discurso político”, Oralia, 17, págs. 375-392. 